\title{
EXTENSIBILITY OF THE HUMAN PULMONARY TRUNK
}

\author{
BY \\ PETER HARRIS, DONALD HEATH, AND ANDREAS APOSTOLOPOULOS \\ From the Departments of Medicine and Pathology, The University of Birmingham, \\ The Queen Elizabeth Hospital, Birmingham 15
}

Received October 28, 1964

The mechanical properties of the walls of blood vessels have an important influence on the flow of blood and passage of pulse waves along them. Though a certain amount of information is available concerning the mechanical properties of the aorta and its main branches (Burton, 1954), we have been unable to find any observations on the pulmonary circulation. Hence, in view of the increasing interest in human pulmonary hæmodynamics, it seemed worth while to investigate the physical characteristics of the wall of the pulmonary trunk in man.

\section{SUBJECTS AND METHODS}

Observations were made at necropsy on 48 patients in whom there was no cardiac or pulmonary disease. A circumferential strip, $1 \mathrm{~cm}$. in width, was cut from the pulmonary trunk $1 \mathrm{~cm}$. above the level of the pulmonary valve by means of a rectangular punch operated by means of a simple lever. The loose adventitial connective tissue was stripped off and each end of the strip placed in a serrated clamp (A in Fig. 1), tightened by means of a screw. One clamp was fixed to a metallic rod; this was attached to a cross bar (B) which could be moved by means of a screw (C) and two parallel runners (D) upwards and downwards in a bath filled with physiological saline and maintained at $37^{\circ} \mathrm{C}$. The other clamp was suspended vertically above the first by means of a nylon thread attached to one arm of a balance. The opposite arm of the balance acted as a pointer along a circular scale which indicated directly the weight that had been applied to the strip of artery. The scale was calibrated by the application of a series of standard weights for a particular adjustment of the counterweights $E$ and $F$. Thus, by turning the screw, the strip of artery was subjected to an increasing extensile load, the magnitude of which could be read off on the scale of the balance.

The length of the strip at any load was measured by a telescopic optical system which moved along a vertical scale. A hair-line within the optical system was aligned first with the clamp at one end of the strip of artery and then with the other. The distance between the two clamps could thus be measured along the vertical scale which could be read to $0.01 \mathrm{~cm}$. by means of a micrometer adjustment and a Vernier scale. The length of the arterial strip was measured first in the unstretched state and then with extensile loads of 10, 20, 40,60, and $100 \mathrm{~g}$. weight, successively. Five minutes were allowed between the application of an increment in load and the measurement of the degree of extension. During this time a certain amount of slow elongation of the strip occurred while the load was maintained constant by adjusting screw $\mathrm{C}$.

In 30 specimens the extensile load was reduced to zero immediately after the final measurement at $100 \mathrm{~g}$. weight. After a further 5 minutes, the length of the strip was again measured, and it was found to average 3.0 per cent greater than the original length. The original length of the strip between the clamps varied between 2.06 and $4.18 \mathrm{~cm}$., the average being $3.29 \mathrm{~cm}$. In view of the variation in initial length, the degree of extension has been expressed as a percentage of the unstretched measurement.

After these physical measurements had been made, the strip was removed from the bath and fixed in formol saline for 48 hours. The strip of artery was embedded in paraffin wax by the usual histological technique, and sections were prepared so that the microtome knife edge cut the long axis of the arterial 


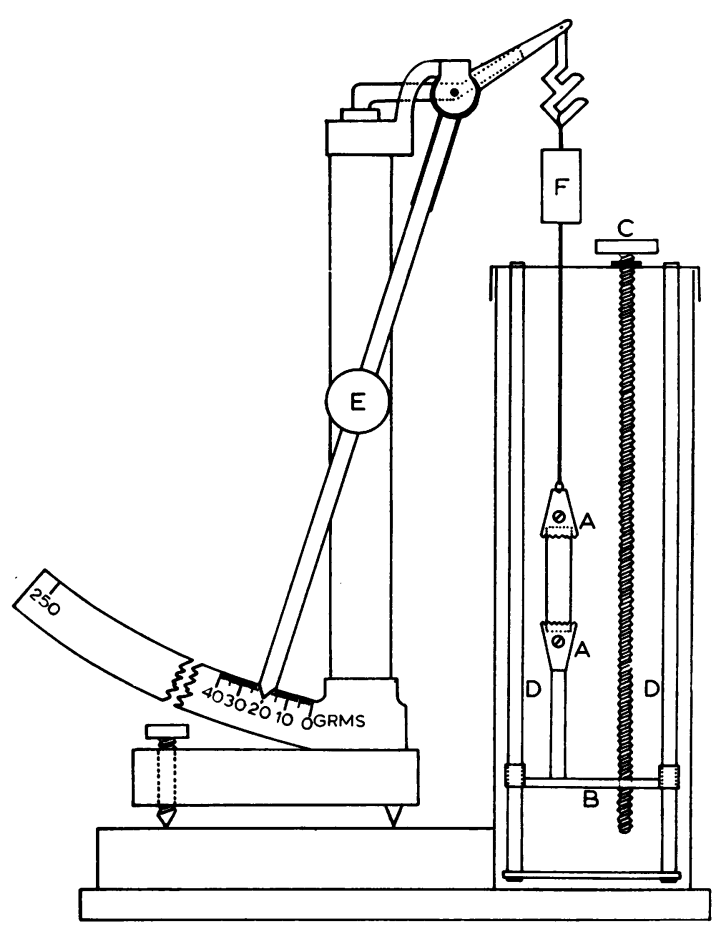

FIG. 1.-Diagram of apparatus. The letters are referred to in the text. strip. It was found that sections cut at right angles to this, necessitating the passage of the knife edge through the entire length of the strip, produced distortion and kinking of the media with considerable variation in medial thickness. Thin sections $(5 \mu)$ were stained by the Lawson modification of the Weigert-Sheridan method for elastic tissue and counterstained with van Gieson's reagents to demonstrate collagen and muscle fibres.

As a result of the careful removal of adventitial tissue before stretching the arterial strip, there were, in almost all cases, practically no collagen fibres external to the media. When such fibrous fragments were present they were not included in the measurement of medial thickness. Some of the strips showed acellular intimal fibrosis due to age. It was impossible to remove such non-medial collagen before stretching the arteries, and the thickness of such intimal plaques, usually focal and of insignificant dimensions compared to that of the media, was included in the measurement of medial thickness. Acid mucopolysaccharides in the media were not stained specifically so that we do not know if minor excesses of ground substance were present. There was, however, no evidence of cystic medial necrosis in any of the arterial strips included in the study.

Initially an attempt was made to measure the thickness of the media in the fresh specimens, but this was found to be so inaccurate that it was not continued. The medial thickness was instead measured on the section of fixed, stained tissue, the figure arrived at being a mean value calculated from usually about 20 measurements made at intervals along the length of the strip. Since the histological techniques caused a certain amount of shrinkage of the tissue, these measurements give an erroneously low value. In a group of five strips in which measurements of thickness of the fresh specimens were made with great care the degree of shrinkage was $24,31,37,26$, and 25 per cent. In the following calculations, the original measurements of thickness of the stained section are used, since their individual accuracy is more certain and the main intention of the study has been to compare different age-groups. Multiplication by a factor of $0 \cdot 7$ will, however, convert the calculation of force per unit area to more absolute figures should this be desired.

All the subjects included in this study were over the age of 2 years and had lived at low altitudes all their lives so that all the arterial strips exhibited the 'adult pulmonary' configuration of elastic tissue (Heath et al., 1959) (Fig. 2). We include the factor of altitude because recent studies by Saldaña and Arias-Stella (1963) have shown that such a state of affairs would not be met with in subjects living at high altitudes. In all the specimens we studied, the elastic tissue was more irregular and sparse than in the aorta. The elastic fibrils were widely spaced and many of them were bizarre in form with club-like terminal expansions (Fig. 2A). Many were short and fenestrated and were associated with intervening slender fibrils. Branching of individual fibrils in all directions was common. Usually, a thick, well-defined, internal elastic lamina was present. It will be noted that the major characteristics of the elastic tissue pattern of the aortic media were absent. That is, the elastic fibrils were not long, numerous, uniform, or packed tightly together and they did not run parallel with one another. Muscle fibres were readily identifiable, especially in the arterial strips from younger subjects, by their characteristic tinctorial properties with van Gieson's stain. Collagen fibres could be demonstrated in the media of pulmonary trunk of even the youngest subjects studied, but there was a considerable increase of fibrosis of the media with increasing age (Fig. 2 and 3). This was so pronounced in the pulmonary trunk of the aged that it stained predominantly red with van Gieson's stain. At the same time there appeared to be degeneration of the elastic fibrils with changes in their staining properties (Fig. 2B). The internal elastic lamina was still recognizable but there was commonly elastosis. There was some clumping of amorphous masses of elastic tissue in the media. In some arterial strips from 


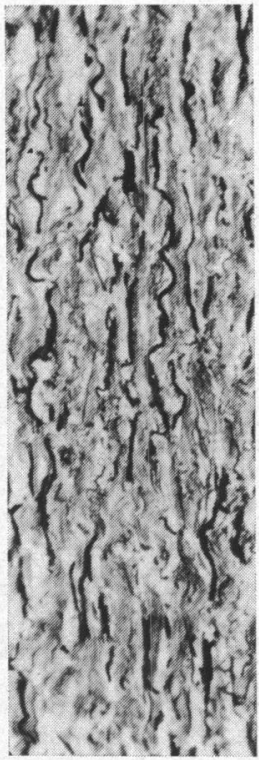

A

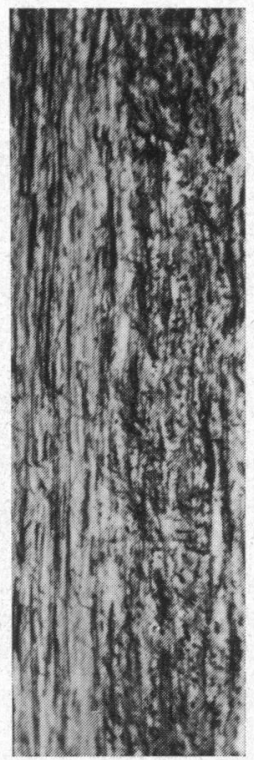

B

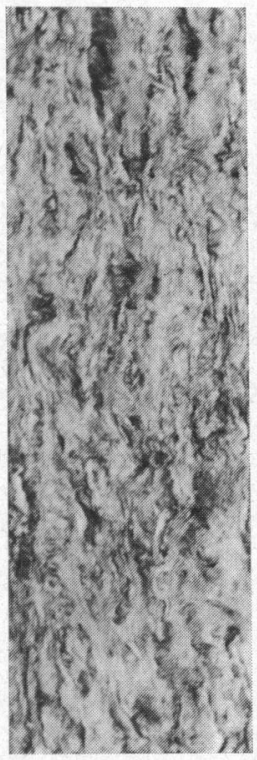

A

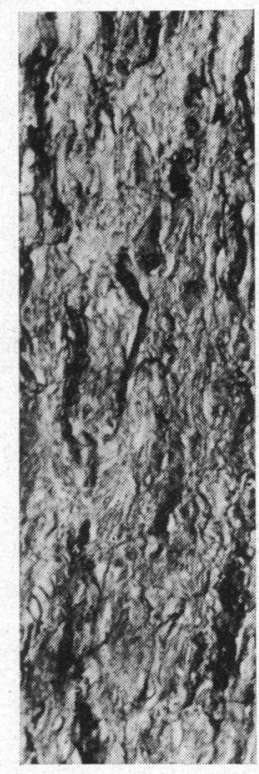

B
Fig. 2.-Sections of pulmonary trunk stained to demonstrate elastic tissue by the Lawson modification of the Weigert-Sheridan method, and counterstained with van Gieson's stain to demonstrate muscle and collagen.

A Man aged 34 years. The branched elastic fibrils are widely spaced and irregular in thickness. (x150.)

B Woman aged 87 years. The elastic fibrils show degeneration with much loss of their characteristic tinctorial properties. There is a considerable excess of fibrous tissue in the media. $(\times 150$.
FIG. 3.-Sections of pulmonary trunk stained with Mallory's stain to demonstrate muscle and collagen. The fibrous tissue shows up black in the photographs,

A Boy aged 7 years. Collagen fibres sparse. $(\times 150$.)

B Man aged 87 years. Collagen fibres plentiful. ( $\times 150$.

these aged subjects there appeared to be loss of muscle fibres with falling together of the degenerate elastic fibrils which showed fuzziness on staining (Fig. 2B). The acellular intimal fibrosis referred to previously also tended to be more pronounced with increasing age.

\section{RESULTS}

The extensile force is expressed in dynes per mm. ${ }^{2}$ mean cross-sectional area of the strip. In the unstretched state, the mean cross-sectional area has been calculated as the width of the strip $(10 \mathrm{~mm}$.) multiplied by the average thickness. However, when extension was applied, the lateral borders of the strip became concave, and the mean cross-sectional area has been calculated by assuming that the original volume of tissue remained unchanged. Hence, the mean cross-sectional area at any degree of stretch was given by:

$$
\frac{\text { Original length } \times \text { original thickness } \times \text { original width }}{\text { Stretched length }} .
$$

The relation between length and extensile force in a circumferential strip of the pulmonary trunk is shown in Fig. 4. The relation is a curved one so that there is a diminishing degree of extensibility with an increasing force. For comparison the figure also shows the results of similar observations on a circumferential strip of the ascending aorta from the same subject. The extensibility of the pulmonary trunk was compared with that of the aorta in 9 other subjects, and the results 


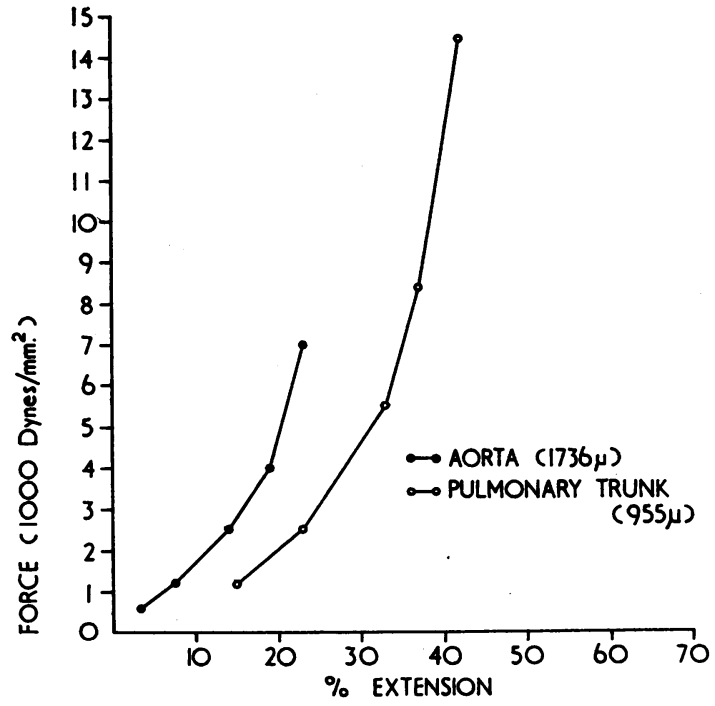

Fig. 4.-A comparison of the extensibility of the pulmonary trunk and aorta in a woman aged 65 years.

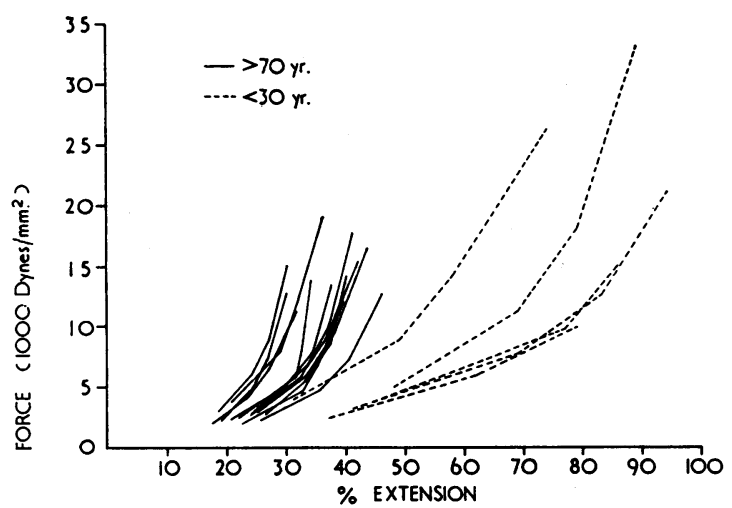

FIG. 5.-The extensibility of the pulmonary trunk in subjects dying under 30 and over 70 years of age.

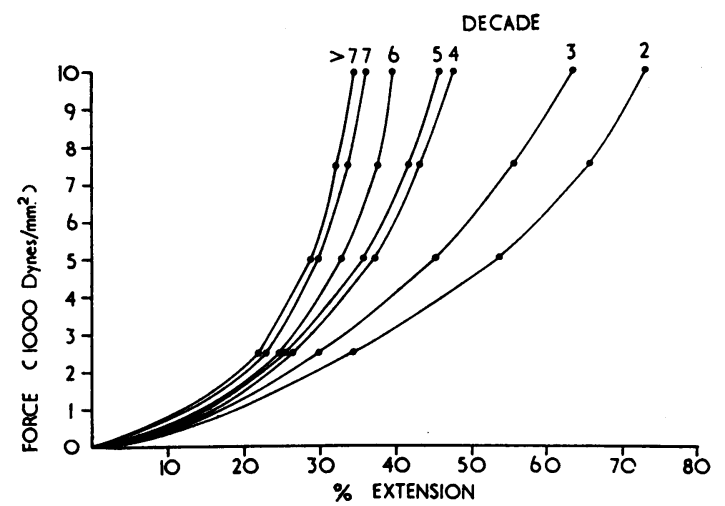

FIG. 6.-Average values for the extensibility of the pulmonary trunk at different decades.

TABLE I

Average Values for Percentage Extension at Various Degrees of Extensile Force Among DifFERENT AGE-GROUPS

\begin{tabular}{c|c|c|c|c|c|c|c|c}
\hline & & & \multicolumn{4}{|c|}{ Percentage extension at different extensile forces } \\
$\begin{array}{c}\text { Age- } \\
\text { group }\end{array}$ & $\begin{array}{c}\text { Age } \\
\text { range }\end{array}$ & $\begin{array}{c}\text { No. of } \\
\text { cases }\end{array}$ & $\begin{array}{c}\text { Mean } \\
\text { age }\end{array}$ & $\begin{array}{c}1000 \\
\text { dynes/mm.2 }\end{array}$ & $\begin{array}{c}2500 \\
\text { dynes/mm.2 }\end{array}$ & $\begin{array}{c}5000 \\
\text { dynes/mm.2 }\end{array}$ & $\begin{array}{c}7500 \\
\text { dynes/mm.2 }\end{array}$ & $\begin{array}{c}10,000 \\
\text { dynes/mm.2 }\end{array}$ \\
\hline $11-20$ & $17-20$ & 3 & $18 \cdot 3$ & $18 \cdot 3$ & $34 \cdot 5$ & $53 \cdot 8$ & $65 \cdot 8$ & $73 \cdot 6$ \\
$21-30$ & $21-25$ & 2 & $23 \cdot 0$ & $17 \cdot 5$ & 29.9 & $45 \cdot 6$ & $56 \cdot 2$ & $64 \cdot 0$ \\
$31-40$ & $31-39$ & 3 & $36 \cdot 3$ & $12 \cdot 8$ & $26 \cdot 6$ & $37 \cdot 4$ & $43 \cdot 4$ & $48 \cdot 1$ \\
$41-50$ & $41-50$ & 9 & $45 \cdot 9$ & $14 \cdot 2$ & $25 \cdot 6$ & $35 \cdot 8$ & $41 \cdot 9$ & $45 \cdot 8$ \\
$51-60$ & $52-59$ & 6 & $55 \cdot 8$ & $14 \cdot 1$ & $24 \cdot 8$ & $33 \cdot 3$ & $37 \cdot 9$ & $40 \cdot 1$ \\
$61-70$ & $61-70$ & 14 & $66 \cdot 0$ & $13 \cdot 9$ & $22 \cdot 8$ & $30 \cdot 0$ & $34 \cdot 0$ & $36 \cdot 6$ \\
$71+$ & $71-88$ & 11 & $76 \cdot 5$ & $13 \cdot 4$ & $22 \cdot 1$ & $29 \cdot 0$ & $32 \cdot 5$ & $34 \cdot 7$ \\
\hline
\end{tabular}


were similar. The tissue of the pulmonary trunk is thus shown to be more extensible than that of the aorta.

In Fig. 5 is shown the relation between length and extensile force in subjects dying under 30 and over 70 years of age. The over-all extensibility of the younger group is evidently greater than that of the older group. The average curves for each decade are shown in Fig. 6 and the data are summarized in Table I. It is again evident that extensibility decreases with age.

The calculation of these average values presented some difficulty. Although the weights applied were the same in each case, the differences in the mean cross-sectional area caused variations in the resulting extensile force per unit cross-sectional area. Hence, in view of the non-linear relation between length and tension, it was not valid to compare the simple arithmetical mean values for extension and extensile force at any given load. Instead, the length-force curve was drawn for each case and the degree of extension for a given extensile force was estimated by interpolation. Average degrees of extension were then calculated for a series of common extensile forces.

The relation between age and the degree of extension for extensile forces of 2500 and 10,000 dynes $/ \mathrm{mm} .^{2}$ is plotted in Fig. 7. The relation evidently becomes more curved and shifts to the right with an increasing extensile force. When age was plotted against the logarithm of the degree of extension, as in Fig. 8, the relation appeared to be a straight line for a given extensile force. Similar relations between age and extension were observed for other degrees of extensile force. Thus, for a particular extensile force, the relation between age and extension could be given by

$$
\Delta l=a e^{k \times \text { age }}
$$

where $\Delta l$ is the percentage increase in length and $a$ and $k$ are constants.

A number of regression lines for $\Delta l$ on age at different values of extensile force were calculated, and they are shown in Fig. 9. Hence this figure may serve as a nomogram for the prediction of the relation between length and extensile force at a given age.

The values of the constants $a$ and $k$ varied according to the magnitude of the extensile force $(F)$. They could be derived from the regression equations relating $\log _{e} \Delta l$ to age and a number of values

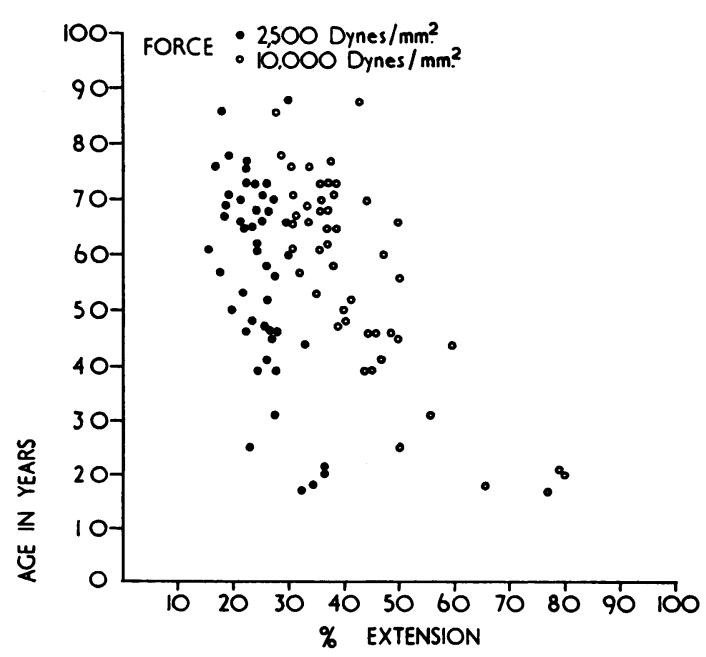

FIG. 7.-The relation between age and percentage extension at extensile forces of 2500 and 10,000 dynes/mm.2.

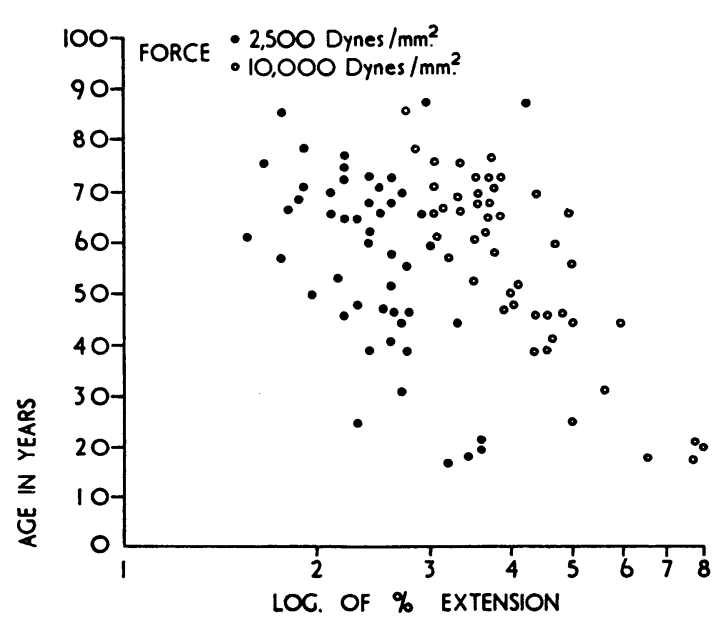

FIG. 8.-The relation between age and the logarithm of the percentage extension at extensile forces of 2500 and 10,000 dynes/mm. ${ }^{2}$ 
TABLE II

Values for $a$ ANd $k$ for Different Degrees of Extensile Force, Calculated from the Regression EQUATON $\Delta l=a e^{k \times \text { age }}$

\begin{tabular}{c|c|c}
\hline $\begin{array}{c}\text { Extensile force (F) } \\
\text { (dynes/mm. }{ }^{2}\end{array}$ & $a$ & $k$ \\
\hline 1,000 & $16 \cdot 3$ & -0.00301 \\
2,500 & $33 \cdot 4$ & -0.00588 \\
5,000 & $49 \cdot 3$. & -0.00832 \\
7,500 & $66 \cdot 6$ & -0.00998 \\
10,000 & $75 \cdot 8$ & -0.01094 \\
12,500 & $78 \cdot 6$ & -0.01074 \\
15,000 & 88.9 & -0.01250 \\
\hline
\end{tabular}

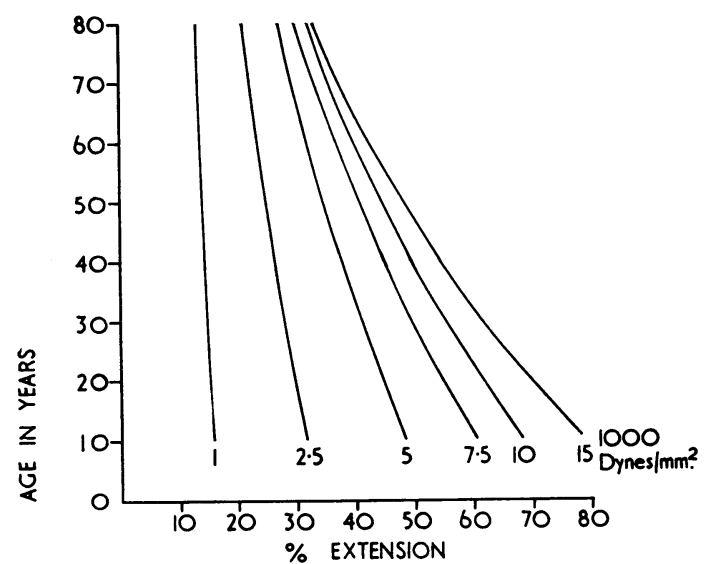

FIG. 9.-Regression lines for percentage extension on age at different values of extensile force.

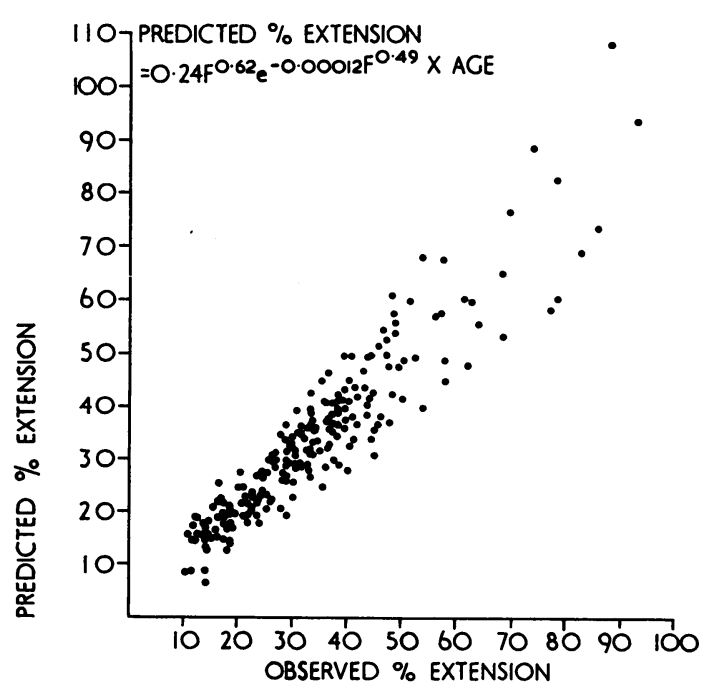

FIG. 10.-The relation between the predicted and observed values for percentage extension.

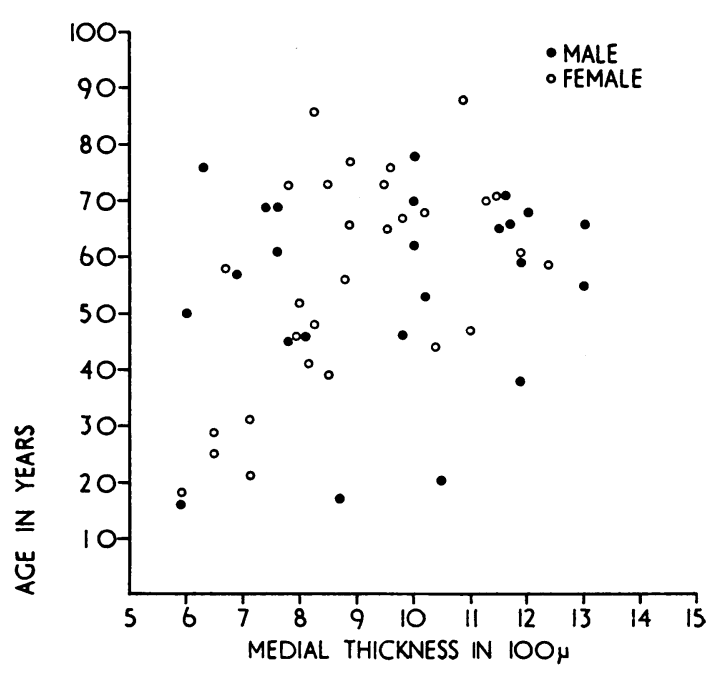

FIG. 11.-The relation between age and medial thickness of the pulmonary trunk in the two sexes.

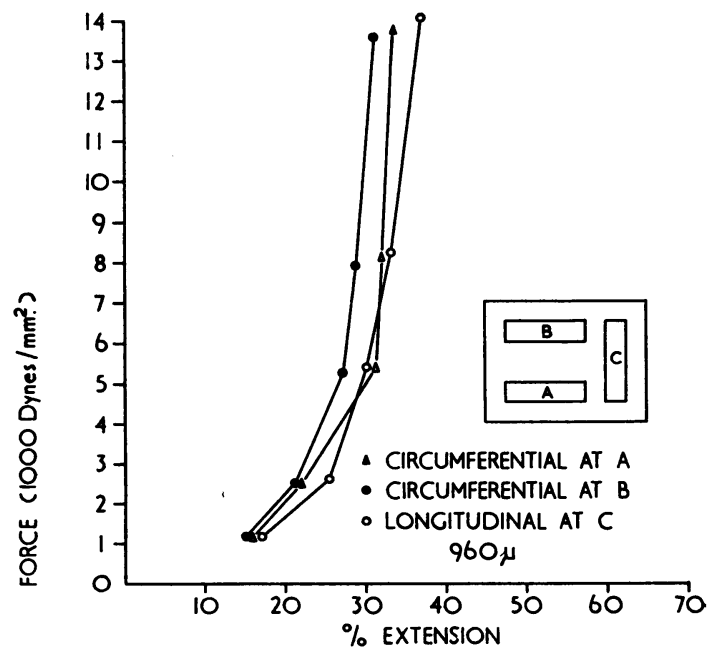

Fig. 12.-A comparison of the extensibility of circumferential and longitudinal strips of the pulmonary trunk taken from the same subject. 
are given in Table II. The relation between $\log _{\mathrm{e}} F$ and $\log _{\mathrm{e}} a$ was approximately a straight line $(r=+0.9927)$ described by the regression equation:

$$
a=0 \cdot 24 F^{0.62}
$$

Similarly the relation between $\log _{\mathrm{e}} F$ and $\log _{\mathrm{e}} k$ was approximately a straight line $(r=+0.9748)$ described by the regression equation:

$$
k=-0 \cdot 00012 F^{0 \cdot 49}
$$

The substitution of equations (2) and (3) in equation (1) thus gives a composite expression of the relation between the extensile forces, the degree of extension, and the age of the individual:

$$
\Delta l=0.24 F^{0.62} e^{-0.00012 F^{0.49} \times \text { age }}
$$

Fig. 10 plots the degree of extension against that predicted by equation (4) for all observations.

Differences in sex were not distributed uniformly throughout the various age-groups. The use of equation (4), however, makes it possible to examine the effect of sex on the extensibility of the pulmonary trunk irrespective of age. There were 17 male subjects in whom the same 5 extensile loads had been applied. The 17 mean observed values for percentage extension in these subjects were less than the predicted values by an average of 0.36 (S.D. 4.54). Among the 28 women in whom there had been a similarly uniform experimental procedure, the observed values exceeded the predicted values by an average of $0 \cdot 25$ (S.D. 4.32). These differences could readily have arisen by chance. The thickness of the strips of pulmonary trunk did not vary according to age or sex (Fig. 11).

The possibility of variations in the extensibility of strips taken from different places in the pulmonary trunk was investigated in one subject. Fig. 12 shows length-force diagrams from three strips of the pulmonary trunk, one longitudinal and two circumferential. The extensibility of all these strips was very similar. The longitudinal strip was slightly more extensible than the circumferential strips, but it does not seem likely that this difference is significant.

\section{Discussion}

The curved relation between the degree of extension and the extensile force in the pulmonary trunk is similar to what has been found in systemic arteries (Burton, 1954). The observation that the wall of the pulmonary trunk is more extensible than that of the aorta has been made previously (Harris and Heath, 1962). Part of this difference could be attributed to the greater thickness of the aortic wall, but the present observations show that the tissue of the media of the pulmonary trunk is intrinsically more extensible than that of the aorta. It seems likely that these differences in physical properties are partly related to the greater abundance of elastic tissue in the aorta (Heath et al., 1959). The role of collagen, however, may well be more important.

Examination of the histological sections of the strips of pulmonary trunk confirmed previous observations (Heath et al., 1959) that with advancing age there is a decrease in elastic tissue and an increase in collagen. Fig. 2 and 3 illustrate the difference in structure. Presumably the decreasing extensibility of the pulmonary trunk with advancing age is due to the increase in collagen.

Roach and Burton (1957) ascribed the curved shape of the length-tension diagram of the walls of arteries to a shorter relaxed length of the elastic tissue relative to that of the collagen. Thus the first and more horizontal part of the curve was thought to be due to the extension of elastic tissue alone, while the gradual involvement of the collagenous fibres coincided with the development of the more vertical later part of the curve.

It seems unlikely that all fibres have the same unstretched length and, with increasing extension, one would expect a progressive recruitment of both elastic and collagenous fibres opposing further stretch. Thus, at a particular length of the arterial strip $(l)$ there will be a number of elastic fibres stretched in varying degrees according to their unstretched length $\left(\lambda_{e}\right)$ and a number of collagenous 
fibres stretched according to their unstretched length $\left(\lambda_{\mathrm{c}}\right)$. If $E_{\mathrm{e}}$ and $E_{\mathrm{c}}$ are the coefficients of elasticity of elastic and collagenous fibres respectively, the total extensile force may be represented as

for all fibres, where $\lambda<l$.

$$
-F=\sum E_{\mathrm{e}}\left(l-\lambda_{\mathrm{e}}\right)+\sum E_{\mathrm{c}}\left(l-\lambda_{\mathrm{c}}\right)
$$

Such a representation is naturally an oversimplification, since not all fibres of one type have the same thickness nor is their orientation homogeneous. It also assumes that all the fibres obey Hooke's law. It does, nevertheless, give some indication of the probable nature of the relation

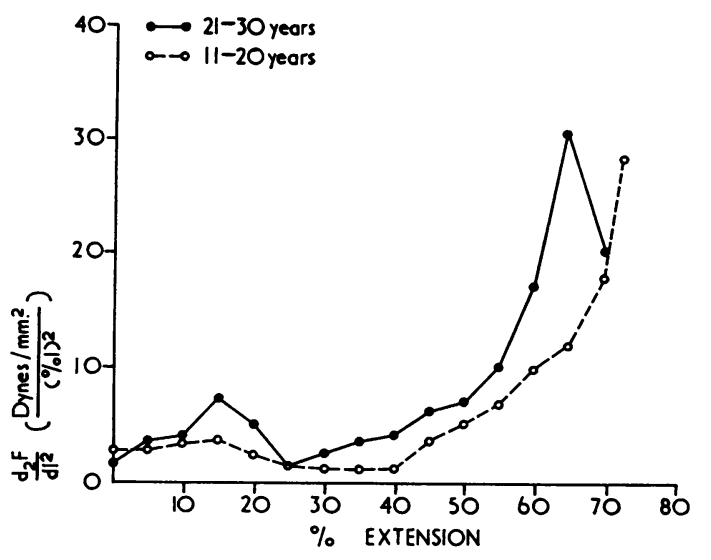

FIG. 13.-The average relation between $\frac{d_{2} F}{d l^{2}}$ and the degree of extension for the second and third decades.

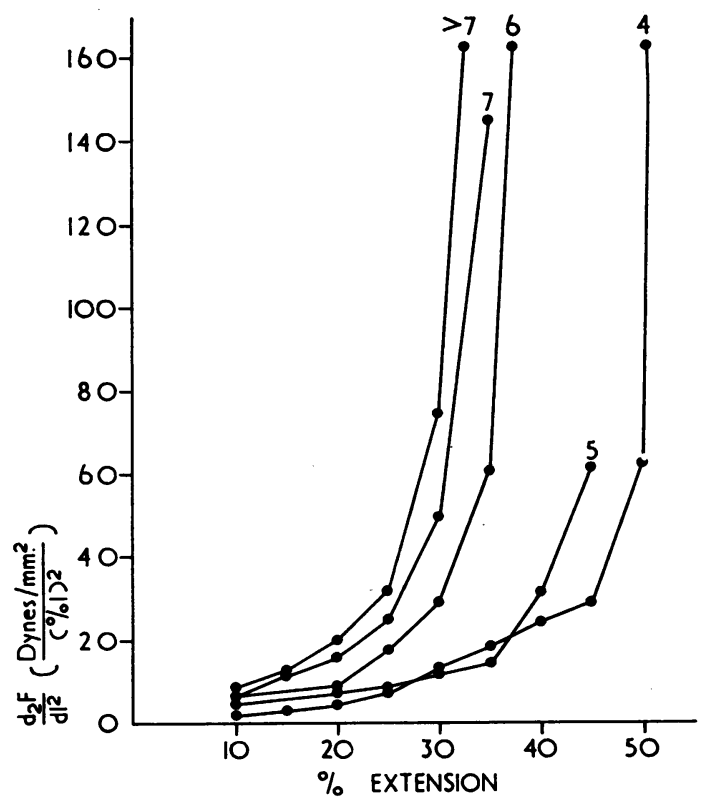

FIG. 14.-The average relation between $\frac{d_{2} F}{d l^{2}}$ and the degree of extension for the fourth and subsequent decades. between the degree of extension and two groups of "elastic forces" due respectively to elastin and collagen.

Thus $\frac{d F}{d l}$ describes the recruitment of new "elastic forces" (whether these be of elastin or collagen) and $\frac{d_{2} F}{d l^{2}}$ describes the rate of recruitment. Curves for $\frac{d_{2} F}{d l^{2}}$ plotted against the degree of extension were derived graphically from the average length-force diagrams for various decades. They are shown in Fig. 13 and 14. In the two youngest groups (Fig. 13) there is a distinct suggestion of the presence of two different populations of elastic forces. Presumably the population arising closer to the unstretched length represents elastic tissue while the second population represents collagen. In the case of the third decade, the graph continues beyond the mode of the second population, but this was not observed in any other group. Such an observation could theoretically be due to a stretching of the fibres beyond their "yield point," but, in view of the high coefficient of elasticity of collagen (Burton, 1954), this seems unlikely. The plots for the fourth and subsequent decades are shown in Fig. 14. They demonstrate a single population of elastic forces, only the beginning of which is included. Presumably this is the collagenous population. Its point of origin shifts progressively towards the unstretched length with increasing age, which suggests either that the population of collagenous forces has become so much greater that its range has spread towards the unstretched point, or that the mean of the population has shifted towards this point. It is possible that the early appearance of the population of collagenous forces in the older age-groups obscures what deflections in the curve might have been due 
to the elastic tissue forces, especially since the histological evidence suggests that the elastic tissue forces become less with advancing age.

How much the chemical changes that accompany death affect the extensibility of the pulmonary trunk remains unknown. Perhaps the most important effect of death is the loss of activity of the muscle fibres which occur in the walls of the pulmonary trunk. Thus the present observations take no account of the role of muscle fibres in the static extensibility of the pulmonary trunk. Neither do they explore the inertial, viscous, and muscular forces which are brought into play during the rapid changes in transmural pressure that, in life, accompany each beat of the heart. Finally it may be noted that the present observations refer only to the properties of the media and the role of the adventitia has not been investigated.

\section{SUMMARY}

The extensibility of circumferential strips of the pulmonary trunk has been examined in 48 cadavers. The relation between force per unit cross-sectional area and extension is a curved one such that extensibility decreases with an increasing extension.

The extensibility also decreases with an increasing age, and this is related to the decrease in elastic tissue and increase in collagen which accompanies ageing. An empirical expression is given for the relation between extensile force, percentage extension, and age.

Analysis of length-force curves gives evidence, in younger subjects, of two distinct groups of elastic forces-one presumably associated with the elastic fibres, and the other with collagen.

\section{REFERENCES}

Burton, A. C. (1954). Relation of structure to function of the tissues of the wall of blood vessels. Physiol. Rev., $34,619$.

Harris, P., and Heath, D. (1962). The Human Pulmonary Circulation; its Form and Function in Health and Disease. Livingstone, Edinburgh.

Heath, D., Wood, E. H., DuShane, J. W., and Edwards, J. E. (1959). The structure of the pulmonary trunk at different ages and in cases of pulmonary hypertension and pulmonary stenosis. J. Path. Bact., 77, 443.

Roach, M. R., and Burton, A. C. (1957). The reason for the shape of the distensibility curves of arteries. Canad. J. Biochem., 35, 681 .

Saldaña, M., and Arias-Stella, J. (1963). Studies on the structure of the pulmonary trunk. II. The evolution of the elastic configuration of the pulmonary trunk in people native to high altitudes. Circulation, 27, 1094. 